Research Article

\title{
AVALIAÇÃO DA INFLUÊNCIA DA INTERFACE SOLO-AR SOBRE OS DADOS COLHIDOS EM POÇOS COM O RADAR POLARIMÉTRICO
}

\author{
Eliezer P. Lopes ${ }^{1} \&$ Elizeu P. Lopes \\ Received August 2, 1999 / Accepted May 25, 2000
}

\begin{abstract}
O objetivo principal deste artigo é determinar o menor valor de profundidade a partir do qual se pode afirmar que os sinais coletados em poços, com o radar geofísico, não são significativamente afetados pela onda lateral e pela onda refletida na interface solo-ar. Esse valor de profundidade é de importância crucial para sondagens eletromagnéticas efetuadas a partir de poços. Apresentam-se três métodos de resolução do problema em questão: um método numérico, um método gráfico e um método analítico. Cinco exemplos práticos reais ilustram a utilidade dos métodos propostos. Destarte, evidenciam-se as vantagens dos métodos sobre a maneira usada classicamente para resolver o mesmo problema. Graças aos métodos desenvolvidos, podem-se evitar as tarefas infrutíferas de coleta e de processamento de muitos dados que serão posteriormente descartados, por estarem significativamente degradados pela interface solo-ar. Os custos envolvidos nessas tarefas podem ser elevados em sondagens eletromagnéticas geotomográficas, realizadas a partir de poços, devido ao grande número de dados tipicamente envolvido nessas sondagens. Demonstra-se, com exemplos práticos reais, que os métodos propostos facilitam o planejamento dessas sondagens eletromagnéticas e a interpretação dos geotomogramas. Se os métodos indicarem que é desprezível a influência da interface solo-ar sobre os dados medidos em poços com o radar geofísico, então os artefatos presentes nos geotomogramas não podem ter sido causados pela interface solo-ar. O plano de incidência contém ambos os poços, onde ficam as antenas. Um dipolo elétrico, localizado em um poço, pode emitir ondas com a polarização transversal magnética (TM). De um poço, podem-se emitir ondas com a polarização transversal elétrica (TE), empregando-se uma antena com uma fenda orientada axialmente em um cilindro condutor. Os métodos desenvolvidos se aplicam a esses dois casos.
\end{abstract}

Palavras-chave: Geoprocessamento; Interface; Poço; Polarimetria; Radar geofísico.

ASSESSING THE INFLUENCE OF THE GROUND-AIR INTERFACE ON POLARIMETRIC BOREHOLE-RADAR DATA - The main purpose of this paper is to determine the minimum depth value beyond which it is possible to state that boreholeradar data are not significantly affected by the lateral wave and the wave reflected at the ground-air interface. This depth value is of paramount importance for crossborehole electromagnetic probing. Three methods of solving the problem in question are presented: a numerical method, a graphical method and an analytical method. Five practical examples illustrate the use of the methods. Thus the advantages of the proposed methods over the classical way of solving the same problem are stressed. Thanks to the new methods, it is possible to avoid the unfruitful tasks of collecting and processing data that will be discarded later, when it is discovered that they are significantly degraded by the ground-air interface. The costs of the unproductive tasks may be high in geotomographic electromagnetic surveys owing to the large amount of data they typically involve. Practical examples show that the proposed methods make easier the planning of such surveys and the interpretation of geotomographs. The cause of artifacts in a geotomograph cannot be ascribed to the ground-air interface when the methods indicate that its influence over the borehole- 
radar data is negligible. The plane of incidence contains both boreholes. An electric dipole, placed in a borehole, can emit transverse magnetic (TM) waves. An axial slot on a conducting cylinder can emit transverse electric (TE) waves from a borehole. The proposed methods apply to both these cases.

Key words: Geoprocessing; Interface; Borehole; Polarimetry; Georadar.

\author{
${ }^{1}$ Climerh - Epagri - Universidade do Sul de Santa Catarina \\ Caixa Postal 951 - 88010-970, Florianópolis, Brasil. \\ eliezer@zaz.com.br
}

\section{INTRODUÇÃO}

A Fig.1 apresenta uma geometria que é muito usada em sondagens que empregam a geotomografia eletromagnética (Lopes, 1989; Lopes \& Lopes, 1989). Quando as antenas estão perto da superfície do solo, não é válido o modelo de propagação de ondas eletromagnéticas que admite que a única trajetória existente entre as antenas transmissora e receptora é a linha reta, conforme mostra a Fig.1. Antenas localizadas perto da superfície do solo podem transmitir ou captar ondas eletromagnéticas que são guiadas pela interface solo-ar. Essas ondas se chamam ondas laterais (Hansen \& Johansen, 2000). Se as antenas transmissora e receptora estiverem em profundidades pequenas, então a onda refletida na interface pode cancelar efetivamente a onda direta, e os sinais recebidos podem ser influenciados principalmente pela onda lateral. As trajetórias dessas três ondas estão mostradas esquematicamente na Fig.2 (Lopes, 1998). Conforme a profundidade das antenas aumente, as ondas direta e refletida deixam de se cancelar, e a influência delas sobre os sinais recebidos se torna importante. Se a profundidade das antenas aumentar ainda mais, a contribuição da onda lateral para os sinais recebidos tende a tornar-se desprezível. Para facilitar a análise da influência da interface solo-ar sobre os sinais medidos, admite-se que o solo seja homogêneo, linear, isotrópico, e que ambas as antenas sejam dipolos elétricos curtos, localizados à mesma profundidade. $\mathrm{Na}$ região de Fraunhofer, os sinais emitidos por qualquer tipo de antena tendem a apresentar características semelhantes às dos sinais emitidos por um dipolo elétrico curto (Lopes, 1998). Os cinco exemplos práticos reais, que serão analisados, demonstram a validade das simplificações teóricas. As simplificações não são suficientemente restritivas, para inviabilizar a utilidade dos resultados da análise a ser apresentada, em vista da natureza aproximada do objetivo estabelecido, que é a determinação da profundidade a partir da qual é desprezível a influência da interface solo-ar sobre os sinais captados. Essa é a principal contribuição deste artigo. Não é do conhecimento dos autores deste trabalho a existência de outros métodos capazes de prever a menor profundidade a partir da qual a influência da interface solo-ar sobre a onda direta é desprezível. Nas sondagens eletromagnéticas efetuadas em poços atualmente, essa influência é avaliada experimentalmente, o que apresenta o inconveniente de requerer a coleta de dados que posteriormente serão descartados das análises, por estarem significativamente degradados pela interface solo-ar (Alumbaugh \& Newman, 1997). O tempo e o custo envolvidos na coleta e no processamento dos dados descartáveis podem ser grandemente reduzidos, graças aos emprego dos métodos apresentados neste trabalho. Essa redução é especialmente significativa em sondagens geotomográficas eletromagnéticas (Lopes, 1989; Lopes \& Lopes, 1989), porque a quantidade de dados diminui substancialmente com a exclusão de algumas posições das antenas nos poços, conforme mostra a Fig.1.

A interpretação dos resultados das sondagens efetuadas com o radar de poços é facilitada pelo conhecimento da influência da interface solo-ar sobre os dados. Tal conhecimento é vital para a interpretação dos geotomogramas gerados por essas sondagens, porque a degradação de parte de um geotomograma pode afetar várias partes dele (Lopes et al., 1998), e uma das causas de artefatos em geotomogramas é a desconsideração, ou a consideração inadequada, da influência da interface solo-ar sobre os sinais medidos 


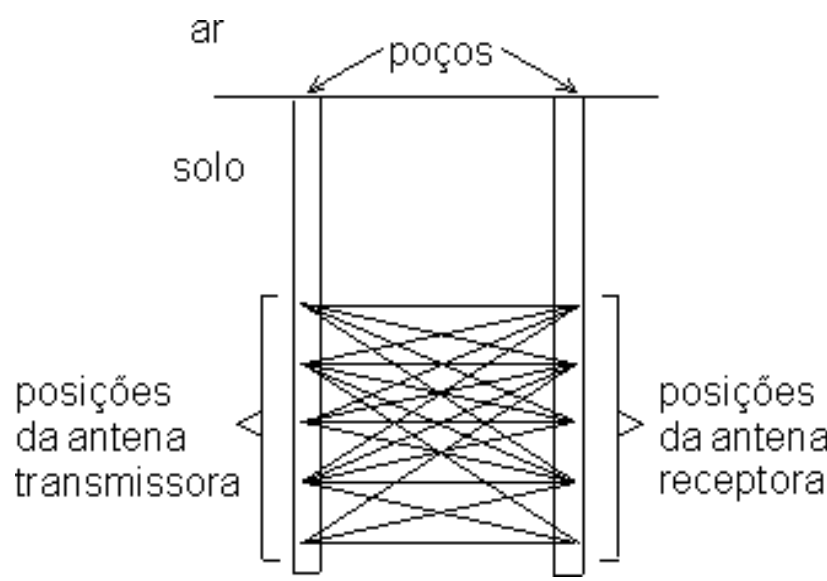

Figura 1 - Uma geometria que permite a sondagem de regiões subterrâneas com o radar geofísico (Lopes \& Lopes, 1992). As antenas estão eletricamente longe da interface solo-ar.

Figure 1 - Cross-hole geometry (Lopes \& Lopes, 1992). The antennas are electrically far from the ground-air interface.

(Ellis \& Peden, 1997). Um dos exemplos analisados neste trabalho (Ramirez, 1986) descreve uma sondagem geotomográfica real, em que se concluiu que os artefatos presentes no geotomograma não podiam ter sido causados pela interface solo-ar, pois a profundidade em que os dados foram coletados era muito grande, para que houvesse qualquer influência significativa da interface solo-ar sobre os sinais captados. Tal conclusão é respaldada pelos métodos propostos. Isso ilustra a importância desses métodos para a interpretação de geotomogramas e para o planejamento de sondagens eletromagnéticas que empregam poços. O estudo da propagação de ondas eletromagnéticas em presença de uma interface entre dois materiais muito diferentes também encontra aplicação na radiodifusão, no projeto de antenas digitais, na monitorização da superfície do oceano por meio de radares costeiros, bem como na comunicação entre submarinos, túneis subterrâneos e poços internos a minas (King \& Brown, 1984; Vesecky, 1999).

Radares geofísicos polarimétricos têm despertado grande interesse, devido à capacidade que eles possuem de fornecer mais informações sobre os alvos do que os radares que empregam apenas um tipo de polarização (Yamaguchi et al., 1995). Na Fig.1, o plano de incidência contém ambos os poços. Um dipolo elétrico, disposto axialmente dentro de um poço, pode emitir ondas que apresentam o campo elétrico contido no plano de incidência. Tais ondas são chamadas de ondas transversais magnéticas (TM). Uma antena com uma fenda orientada axialmente em um cilindro condutor pode emitir, de um poço, ondas que apresentam o campo elétrico polarizado verticalmente ao plano de incidência.
Tais ondas são conhecidas como ondas transversais elétricas (TE). Os métodos apresentados neste trabalho se aplicam a ambos esses casos pelos seguintes motivos. 1) Os métodos foram propositadamente projetados para não dependerem dos coeficientes de reflexão associados às polarizações TM e TE. 2) Os diagramas de radiação associados a ambos os tipos de antenas mencionados são praticamente iguais ao longo do plano de incidência (Kraus, 1992; Miwa et al., 1999; Ramo et al., 1965).

\section{METODOLOGIA}

Em muitas sondagens geotomográficas, utiliza-se a geometria em que os poços apresentados na Fig.1 são paralelos (Lopes \& Lopes, 1994; Miwa et al., 1999). Nesse caso, a intensidade do campo elétrico medido pela antena receptora, na região de Fraunhofer, para a onda que percorreu o caminho direto, indicado na Fig.2, é (King \& Sandler, 1994):

$$
\left|E_{D}^{\prime}\right|=\frac{G f \exp (-\alpha L)}{L},
$$

onde $\alpha$ é o fator de atenuação, $f$ é a freqüência, $L$ é a distância entre as antenas transmissora e receptora e $G$ é um fator que depende principalmente dos

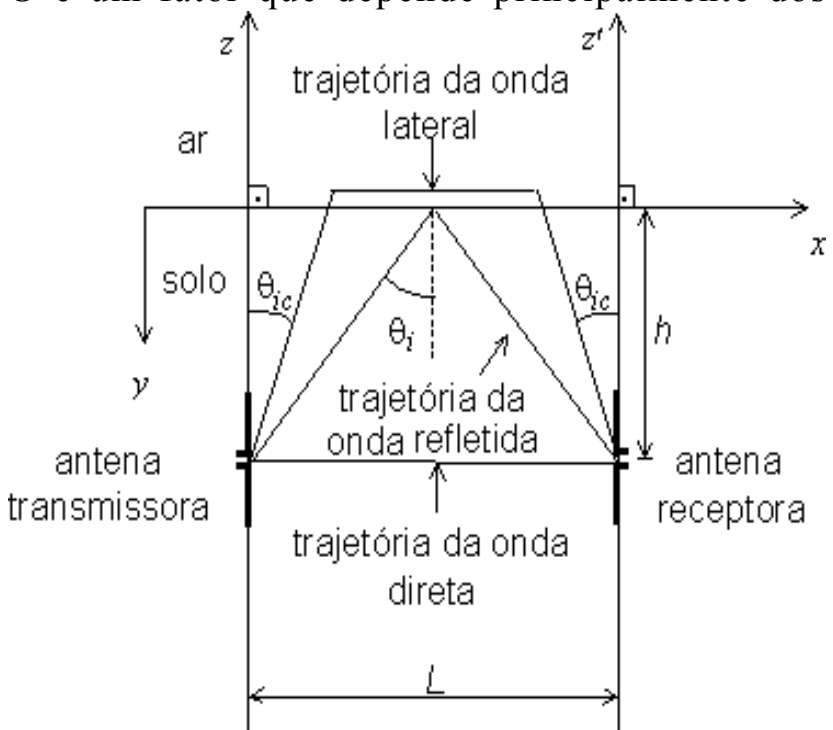

Figura 2 - Trajetórias das ondas eletromagnéticas entre as antenas transmissora e receptora, localizadas perto da interface solo-ar. aDM Admite-se que os eixos $z$ e $z$ ' dos dipolos elétricos curtos sejam perpendiculares à superfície do solo, indicada pelo eixo $x$. $\theta_{i c}$ é o ângulo de incidência crítico (Lopes, 1998).

Figure 2 - Trajectories of the electromagnetic waves between the transmitting antenna and the receiving antenna. The antennas are at shallow depths. The axes $z$ and $z$ ' of the short electric dipoles are perpendicular to the ground surface, which is indicated by the axis x. $\theta_{i c}$ is the critical angle of incidence (Lopes, 1998). 
comprimentos das antenas, da amplitude da corrente elétrica na antena transmissora, das características elétricas das antenas e da forma como for realizado o acoplamento entre as antenas e o meio. $G$ pode ser determinado experimentalmente, através de medições da intensidade do campo elétrico recebido, em um meio onde o fator de atenuação seja conhecido (Alexander \& Salter, 1997). É importante mencionar que $G$ não depende teoricamente das inclinações das antenas nem das orientações das trajetórias da onda direta.

Para a situação apresentada na Fig.2, a intensidade do campo elétrico refletido, medido pela antena receptora, é aproximadamente (Lopes, 1998):

$$
\left|E_{R}^{\prime}\right|=\frac{\left|R\left(\theta_{i}\right)\right| G f \exp \left(\frac{-\alpha L}{\operatorname{sen} \theta_{i}}\right) \operatorname{sen}^{3} \theta_{i}}{L},
$$

onde $\theta_{i}$ é o ângulo de incidência indicado na Fig.2, e $R\left(\theta_{i}\right)$ é o coeficiente de reflexão para a onda plana que apresente o modo transversal magnético, também conhecido como modo TM (King \& Sandler, 1994). Assim, o campo magnético é perpendicular ao plano de incidência, que é o plano $x y$ na Fig.2, e o campo elétrico é paralelo a esse plano. Em um meio dielétrico, o módulo do coeficiente de reflexão é dado por (Kraus, 1992)

$$
\left|R\left(\theta_{i}\right)\right|=\frac{\left|\sqrt{\varepsilon_{r}-\left(\varepsilon_{r} \operatorname{sen} \theta_{i}\right)^{2}}-\cos \theta_{i}\right|}{\left|\sqrt{\varepsilon_{r}-\left(\varepsilon_{r} \operatorname{sen} \theta_{i}\right)^{2}}+\cos \theta_{i}\right|},
$$

onde $\theta_{i}=\operatorname{arctg}\left(\frac{L}{2 h}\right), h$ é a profundidade de ambas as antenas, conforme mostra a Fig.2, e $\varepsilon_{r}$ é a permissividade elétrica relativa média do solo. Dividindo-se a Eq.(2) pela Eq.(1), obtém-se:

$$
Q \triangleq \frac{\left|E_{R}^{\prime}\right|}{\left|E_{D}^{\prime}\right|}=\left|R\left(\theta_{i}\right)\right| \exp \left[\alpha L\left(1-\frac{1}{\operatorname{sen} \theta_{i}}\right)\right] \operatorname{sen}^{3} \theta_{i}
$$

O parâmetro $Q$ quantifica a influência do sinal refletido sobre o sinal de interesse, que é o sinal associado à onda que percorreu o caminho direto. A Eq.(4) é uma ferramenta importante para o projeto de experimentos geotomográficos eletromagnéticos que utilizam poços. Ela permite determinar a profundidade a partir da qual a influência adversa do sinal refletido sobre o sinal de interesse é desprezível. Se
$\widehat{Q} \triangleq \frac{Q}{\left|R\left(\theta_{i}\right)\right|}$, então, a partir da Eq.(4), pode-se escrever (Lopes, 1998)

$$
\widehat{Q}=\exp \left[\alpha L\left(1-\frac{1}{\operatorname{sen} \theta_{i}}\right)\right] \operatorname{sen}^{3} \theta_{i} .
$$

A Eq.(5) possui algumas propriedades que facilitam o projeto do experimento geotomográfico eletromagnético: ela não depende do coeficiente de reflexão, presta-se mais facilmente à investigação analítica do que a Eq.(4) e, geralmente, permite o estabelecimento de uma margem de segurança no projeto, porque o módulo do coeficiente de reflexão só pode assumir valores no intervalo. Se o objetivo do projeto é a determinação da profundidade $h$, a partir da qual $\frac{\left|E_{R}^{\prime}\right|}{\left|E_{D}^{\prime}\right|} \leq \Psi$, onde $0 \leq \Psi \leq 1$, então tal objetivo pode ser alcançado, fazendo-se $\widehat{Q}=\Psi$ na Eq.(5), e resolvendo-se essa equação para $\operatorname{sen} \theta_{i}$. Tal valor de $\operatorname{sen} \theta_{i}$ satisfaz, necessariamente, a condição $Q \leq \Psi$, porque $Q \leq \hat{Q}$. Se o módulo do coeficiente de reflexão, associado ao ângulo $\theta_{i}$ que satisfaz a Eq.(5), for menor do que a unidade, então a profundidade $h$ encontrada é maior do que aquela para a qual $Q=\Psi$. Esse acréscimo no valor da profundidade é a margem de segurança, que garante que a condição $Q \leq \Psi$ não seja violada. Considerações trigonométricas na Fig.2 permitem estabelecer que o relacionamento entre $\operatorname{sen} \theta_{i}$ e a profundidade $h$ das antenas é dado por (Lopes, 1998):

$$
h=\frac{L}{2}\left(\frac{1}{\operatorname{sen}^{2} \theta_{i}}-1\right)^{1 / 2} .
$$

A Fig.3 expressa graficamente a Eq.(5). Apresentamse curvas de $\hat{Q}$ em função do produto $\alpha L$, para diversos valores do seno do ângulo de incidência. $\mathrm{O}$ produto $\alpha L$ pode ser interpretado como a quantidade de profundidades de penetração que a onda direta percorre, quando ambas as antenas estão espaçadas de uma distância $L$. A escala horizontal do nomograma apresentado na Fig.3 é linear. A escala vertical é logarítmica, o que permite a expressão das curvas de 


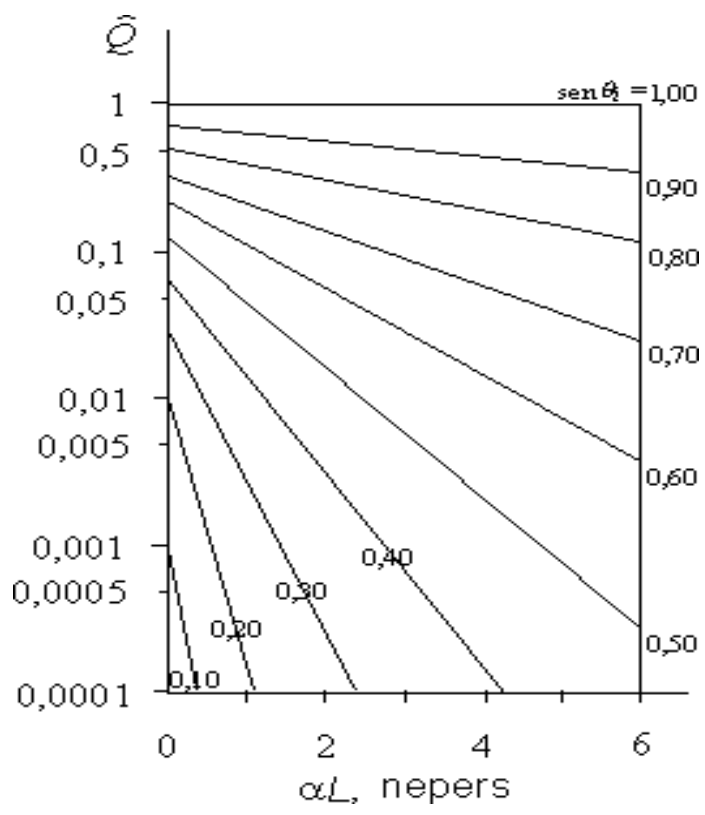

Figura 3 - Expressão gráfica do relacionamento entre $\widehat{Q}$, a quantidade de profundidades de penetração $(\alpha L)$ que a onda eletromagnética percorre ao longo do caminho direto e o seno do ângulo de incidência. $\left|E_{D}^{\prime}\right|$ é a intensidade do campo elétrico recebido, após a onda eletromagnética ter percorrido o caminho direto. $\left|E_{R}^{\prime}\right|$ é a intensidade do campo elétrico recebido, após a onda eletromagnética ter sofrido reflexão na interface solo-ar (Lopes, 1998).

Figure 3 - Graphical representation of the relationship among $\hat{Q}$, the amount of penetration depths $(\alpha L)$ traveled by the electromagnetic wave along the direct path and the sine of the angle of incidence. $\left|E_{D}^{\prime}\right|$ and $\left|E_{R}^{\prime}\right|$ stand for the received intensities of the direct and reflected electric fields, respectively (Lopes, 1998).

$\widehat{Q}$ em função de $\alpha L$ através de linhas retas.

Explicitando-se o $\operatorname{sen} \theta_{i}$, na Eq.(6), obtém-se

$$
\operatorname{sen} \theta_{i}=\frac{L}{\sqrt{L^{2}+4 h^{2}}} .
$$

Essa expressão permite concluir que $\operatorname{sen} \theta_{i} \cong 1$, quando a profundidade $h$ é muito menor do que $L / 2$. A Fig. 3 mostra que $\widehat{Q} \cong 1$, para $\operatorname{sen} \theta_{i} \cong 1$ e para qualquer valor do produto $\alpha L$. Como $Q=\widehat{Q}\left|R\left(\theta_{i}\right)\right|$, então $Q \cong 1$, para valores pequenos de $h$. Conseqüentemente, nesta situação, a Eq.(4) permite que se conclua que $\left|E_{R}^{\prime}\right| \cong\left|E_{D}^{\prime}\right|$. Para a incidência rasante, isto é, para $\theta_{i}=90^{\circ}$, o campo elétrico refletido, medido pela antena receptora, está em oposição de fase ao campo elétrico associado à onda que atravessa o meio geofísico através do caminho direto. Portanto, tais campos se cancelam efetivamente, quando a profundidade das antenas é pequena (Shavit \& Rosen, 1995), seja o solo dispersivo ou dielétrico. O campo elétrico resultante é praticamente causado pela onda lateral. Com o aumento da profundidade das antenas, os campos direto e refletido não mais se cancelam, e a contribuição deles para o campo elétrico total geralmente tende a tornar-se dominante.

Basicamente, os métodos descritos neste trabalho comparam, na recepção, a intensidade do campo elétrico associado à onda que se refletiu na interface solo-ar com a intensidade do campo elétrico relativo à onda que não sofreu reflexões ou refrações significativas, ao longo do trajeto entre a antena transmissora e a antena receptora. A comparação não envolve explicitamente a onda refratada na interface solo-ar porque a intensidade de tal onda costuma ser bem mais fraca do que a intensidade da onda refletida, a partir de determinada profundidade. Algumas causas dessa diferença de intensidade são as seguintes. 1) O diagrama de radiação de um dipolo elétrico curto indica que essa antena emite campos mais intensos na direção perpendicular à direção de seu eixo. As intensidades dos campos diminuem conforme a direção de emissão se aproxime da do eixo do dipolo (Sadiku, 1994). A Fig. 2 mostra que a trajetória da onda refratada é a que mais se aproxima da direção do eixo da antena transmissora (eixo $z$ ). 2) Como o diagrama de radiação da antena receptora foi considerado semelhante ao da antena transmissora, aquela antena atenua mais o sinal associado à onda que a atinja por uma direção mais próxima da direção de seu eixo. A Fig.2 mostra que a onda lateral é a que alcança a antena receptora por uma direção mais próxima da direção do eixo $z$ '.

A Eq.(3) só se aplica a meios dielétricos. Turner \& Siggins (1994) apresentam expressões para os coeficientes de reflexão, válidas para meios dielétricos ou dissipativos, bem como para ondas eletromagnéticas planas que apresentam o modo transversal elétrico (TE) ou o modo transversal magnético (TM). Aplicam-se aos meios dielétricos ou aos meios dissipativos os três métodos apresentados neste artigo, para o cálculo da profundidade a partir da qual se pode afirmar que é 
desprezível a influência da interface solo-ar sobre os sinais captados pela antena receptora. Podem-se avaliar as influências, sobre os sinais recebidos, de outras interfaces que possam haver no meio sondado (Miwa et al., 1999), mediante adaptações simples desses três métodos.

A Eq.(5) mostra que $\widehat{Q}$ depende do fator de atenuação médio do meio $(\alpha)$, da largura da região investigada $(L)$ e do ângulo de incidência da onda eletromagnética na interface solo-ar $\left(\theta_{i}\right) . \alpha$ precisa ser conhecido de antemão, para que se possa avaliar a influência da interface solo-ar sobre os dados associados à onda eletromagnética que percorre o caminho direto entre as antenas transmissora e receptora. Essa avaliação pode ser prejudicada pela escolha de um valor inadequado de $\alpha$. Conseqüentemente, é importante determinar como as incertezas associadas a $\alpha$ influenciam $\widehat{Q}$. Uma definição de sensibilidade é a razão entre a variação percentual na resposta de um sistema e a variação percentual numa grandeza que influencia a resposta (Lopes \& Lopes, 1993). A sensibilidade de $\widehat{Q}$ a $\alpha$ é expressa analiticamente por

$$
\mathrm{S}_{\alpha}^{\hat{Q}}=\frac{\alpha}{\widehat{Q}} \frac{\partial \widehat{Q}}{\partial \alpha}
$$

Substituindo-se a Eq.(5) na Eq.(8), obtém-se

$$
\mathrm{S}_{\alpha}^{\hat{Q}}=\alpha L\left(1-\frac{1}{\operatorname{sen} \theta_{i}}\right)=\ln \left(\frac{\hat{Q}}{\operatorname{sen}^{3} \theta_{i}}\right)
$$

Para pequenas variações percentuais nas vizinhanças do ponto $\left(\alpha_{\circ}, \hat{Q}_{\circ}\right)$, a Eq.(8) permite escrever a aproximação seguinte.

$$
\frac{\Delta \hat{Q}}{\hat{Q}_{\circ}} \cong \frac{\Delta \alpha}{\alpha_{\circ}} \mathrm{S}_{\alpha_{\circ}}^{\widehat{Q}_{\circ}}
$$

onde $\Delta \widehat{Q}=\widehat{Q}-\widehat{Q}_{\circ}$ e $\Delta \alpha=\alpha-\alpha_{\circ}$.

$$
\begin{array}{r}
\text { A Eq.(9) fornece } \mathrm{S}_{\alpha_{\circ}}^{\hat{Q}_{\circ}}=-1,14 \text {, para } \alpha_{\circ}= \\
70^{-1} \mathrm{~Np} / \mathrm{m}, L=168 \mathrm{~m},\left(\operatorname{sen} \theta_{i}\right)_{\circ}=0,6783 \text { e } \widehat{Q}_{\circ}=0,1 .
\end{array}
$$

Os valores dessas quatro grandezas concernem à sondagem efetuada por Lytle et al. (1976), em uma formação predominantemente calcária, localizada no Alasca. Tal sondagem será examinada de maneira mais detalhada no Exemplo 3, que consta da próxima seção. De acordo com a Eq.(10), uma incerteza de $\pm 10 \%$ no valor de $\alpha\left(\Delta \alpha / \alpha_{\circ}= \pm 10 \%\right)$ implica uma incerteza de aproximadamente $\mp 11,4 \%(= \pm 10 \% \times(-$ 1,14)) no valor de $\hat{Q}$.

Desde que a antena receptora esteja em qualquer profundidade $h$ não nula, a Eq.(7) permite concluir que $0<\operatorname{sen} \theta_{i}<1$. Para valores do seno do ângulo de incidência nesse intervalo, o primeiro membro à direita da Eq.(9) indica que a sensibilidade de $\widehat{Q}$ a $\alpha$ é negativa. Esse fato e a Eq.(10) permitem que se conclua que, em qualquer profundidade fixa, $\widehat{Q}$ cresce com a redução de $\alpha$, ou $\widehat{Q}$ decresce com o aumento de $\alpha$.

A negatividade da sensibilidade de $\widehat{Q}$ a $\alpha$, para profundidades não nulas das antenas, aplicada ao segundo membro à direita da Eq.(9), resulta em $\ln \left(\hat{Q} / \operatorname{sen}^{3} \theta_{i}\right)<0$, de onde se deduz que $\ln \hat{Q}<\ln \operatorname{sen}^{3} \theta_{i}$, ou $\operatorname{sen} \theta_{i}>\hat{Q}^{1 / 3}$.

Essa conclusão é útil para estabelecer o valor inicial do seno do ângulo de incidência, que é necessário para resolver numericamente a Eq.(5).

\section{EXEMPLOS PRÁTICOS REAIS}

Nesta seção, apresentam-se cinco exemplos de utilização da metodologia apresentada na seção anterior. No Exemplo 1, ilustra-se o funcionamento do método gráfico e do método numérico de avaliação da influência da interface solo-ar sobre os dados colhidos em poços com o radar geofísico. No Exemplo 2, introduz-se o método analítico, para determinar a profundidade a partir da qual a influência da onda refletida sobre a onda direta pode ser considerada desprezível. O objetivo principal do Exemplo 3 é ilustrar, de forma pragmática, a confiabilidade dos métodos propostos. No Exemplo 4, demonstra-se, principalmente, a utilidade da Eq. (11), tanto para o planejamento de sondagens geotomográficas eletromagnéticas que utilizam poços, como para a interpretação dos resultados dessas sondagens. O Exemplo 5 ilustra, entre outros aspectos, uma maneira de obter in situ o valor do fator de atenuação, que é requisitado pelos três métodos propostos neste trabalho. 


\section{Exemplo 1}

Côte et al. (1995) empregaram a geotomografia eletromagnética para sondar uma região calcária na cidade de Marvejols, localizada na região central da França. A separação entre os poços era igual a $12 \mathrm{~m}$. O texto seguinte foi extraído do artigo de Côte et al. (1995). "Devido aos efeitos da superfície, as medidas podem ser usadas a partir da profundidade de $5 \mathrm{~m}$." Grande parte da imagem reconstruída da região sondada apresenta valores de fator de atenuação próximos a $0,5 \mathrm{~Np} / \mathrm{m}$. Portanto, $\alpha L=0,5 \mathrm{~Np} / \mathrm{m}$ X $12 \mathrm{~m}$ =6. A largura da região é maior do que uma profundidade de penetração. Marca-se o ponto $(\alpha L ; \widehat{Q})=(6 ; 0,1)$ no nomograma apresentado na Fig.3, e conclui-se que $\operatorname{sen} \theta_{i} \cong 0,78$. A Eq.(6) indica que a profundidade correspondente a esse valor do ângulo de incidência é $4,7 \mathrm{~m}$. Outro método de resolução deste problema se baseia na resolução numérica da Eq.(5) para o seno do ângulo de incidência, empregando a expressão (11), para fornecer uma boa estimativa inicial da solução da Eq.(5). Para que a amplitude da onda refletida na interface solo-ar seja, no mínimo, 10 vezes menor do que a amplitude da onda direta, utiliza-se $\widehat{Q}=0,1$ na Eq.(5). A Eq. (11) fornece $\operatorname{sen} \theta_{i}>0,46$. Emprega-se 0,47 como a estimativa inicial do valor do seno do ângulo de incidência, necessária para a resolução numérica da Eq.(5), e conclui-se que $\operatorname{sen} \theta_{i}=0,79$. Utilizando-se a Eq.(6), obtém-se $\mathrm{h} \cong 4,8 \mathrm{~m}$. Tanto o método gráfico quanto o método numérico fornecem resultados próximos a $5 \mathrm{~m}$, que é a profundidade até a qual Côte et al. (1995) notaram a existência de influências significativas da interface solo-ar sobre os dados medidos por eles. A boa concordância entre os valores de profundidade previstos pelos métodos descritos neste trabalho e o valor observado na situação prática real é evidência da adequação de tais métodos à realidade geofísica. Tal adequação tende a substanciar as considerações teóricas efetuadas. Os métodos propostos neste trabalho permitem poupar o tempo e o custo envolvidos na tarefa infrutífera de coletar dados que serão descartados da inversão tomográfica, por estarem degradados pela interface solo-ar. A inclusão desses dados no processo de reconstrução de imagens pode causar o aparecimento de artefatos em diversas partes do geotomograma, principalmente em sua parte superior. O estudo da propagação da informação em problemas inversos geotomográficos, efetuado por Lopes (1998), explica por que, nesse caso, os artefatos não se restringem à parte superior do geotomograma.

\section{Exemplo 2}

Turner \& Siggins (1994) realizaram experimentos geotomográficos com o radar, utilizando antenas localizadas em dois poços paralelos, existentes em uma formação granítica muito desgastada e fraturada. A distância entre os poços era igual a $8,5 \mathrm{~m}$. A análise espectral dos pulsos recebidos indicou que o sinal que sofreu reflexão na interface solo-ar devia apresentar amplitude aproximadamente igual a um décimo da amplitude do sinal que percorreu o caminho direto, quando a antena transmissora se encontrasse à profundidade de $4,8 \mathrm{~m}$ e a antena receptora ficasse à profundidade de $6,1 \mathrm{~m}$. Emprega-se a seguir a metodologia apresentada na seção anterior, para determinar aproximadamente a profundidade a partir da qual se pode dizer, com relativa segurança, que a amplitude do sinal refletido na interface solo-ar é menor ou igual a $10 \%$ da amplitude do sinal que percorreu o caminho direto entre as antenas transmissora e receptora. Consultando-se a literatura sobre as características elétricas de materiais litológicos (Lopes, 1989; Lopes, 1998; Ramirez, 1986), conclui-se que $\alpha \approx 0,1 \mathrm{~Np} / \mathrm{m}$. Portanto, $\alpha L=0,85$. Embora o valor do seno do ângulo de incidência possa ser encontrado através do nomograma apresentado na Fig.3, resolvese inicialmente esse problema por outro método, que é mais simples, bem como mais preciso. Para $\widehat{Q}=0,1 \mathrm{e}$ $0,5 \leq \alpha L \leq 9,8$, várias simulações computacionais indicaram que a seguinte aproximação da Eq.(5) conduz a resultados satisfatórios (Lopes, 1998):

$$
\operatorname{sen} \theta_{i}=0,5928(\alpha L)^{0,1589}+e,
$$

onde $e$ representa o erro associado a essa aproximação. As simulações permitiram concluir que $|e| \leq 0,007$. Desprezando-se esse pequeno erro, e substituindo-se a Eq.(12) na Eq.(6), obtém-se:

$$
\hat{h}=L\left[0,7114(\alpha L)^{-0,3178}-0,25\right]^{1 / 2},
$$

onde $\hat{h}$ é a profundidade associada a $\widehat{Q}=0,1$. Cabe ressaltar que as Eqs.(12) e (13) são válidas para $0,5 \leq \alpha L \leq 9,8$. Essa faixa de quantidades de profundidade de penetração é mais ampla do que a exposta na abcissa do nomograma apresentado na Fig.3. 
Além dessa qualidade, a Eq.(13) permite maior facilidade no cálculo da profundidade a partir da qual $Q \leq 0,1$, em comparação com o uso do nomograma ou com a inversão numérica da Eq.(5), seguidos do emprego da Eq.(6). Para $\alpha L=0,85$ e $L=8,5 \mathrm{~m}$, a Eq.(13) fornece $\hat{h}=6,0 \mathrm{~m}$. A inversão numérica mencionada conduz a $\hat{h}=6,1 \mathrm{~m}$. O valor de profundidade calculado pelo método gráfico é $6,5 \mathrm{~m}$. $\mathrm{O}$ valor de profundidade da antena receptora, relatado por Turner \& Siggins (1994), é 6,1 m. Tal valor concorda bastante bem com os valores calculados pelos três métodos propostos.

\section{Exemplo 3}

Lytle et al. (1976) realizaram sondagens eletromagnéticas com o emprego de dois poços perfurados na localidade de Brooks Range, que fica no norte do Alasca. A região sondada apresentava formação predominantemente calcária. A distância entre os poços era igual a $168 \mathrm{~m}$. As medições efetuadas indicaram que a profundidade de penetração era tipicamente igual a $70 \mathrm{~m}$. Lytle et al. (1976) relatam que a profundidade de penetração média da região investigada decrescia monotonamente com o aumento da freqüência. Eles mencionam que tal comportamento é coerente com o de testemunhos geofísicos. Como o fator de atenuação é o recíproco da profundidade de penetração, conclui-se que $\alpha=70^{-1} \mathrm{~Np} / \mathrm{m}$, no caso analisado. O número de profundidades de penetração é $\alpha L=70^{-1} \mathrm{~Np} / \mathrm{m}$ X $168 \mathrm{~m}=2,4$. Nesta situação, a

Eq.(13) fornece $\hat{h}=90,3 \mathrm{~m}$. Extraiu-se o trecho seguinte do artigo de Lytle et al. (1976). "Conforme as profundidades das antenas aumentem ainda mais, os modos lateral e refletido se tornam irrelevantes em comparação com o modo direto, portanto é razoavelmente válido basear os resultados puramente em uma onda direta. A profundidade em que esse ponto foi atingido no meio investigado foi aproximadamente igual a 91 m." A utilização do método gráfico e a resolução numérica da Eq.(5), seguidas do emprego da Eq.(6), também conduzem a valores de profundidade bem próximos de $91 \mathrm{~m}$. A excelente concordância dos valores de profundidade teoricamente previstos com o valor experimentalmente determinado é forte evidência de que os três métodos desenvolvidos podem ser confiantemente empregados para auxiliar a interpretação dos resultados de sondagens eletromagnéticas, realizadas a partir de dois poços, conforme mostrado na Fig. 1. Podem-se assim identificar os resultados que mais fidedignamente retratam as características do meio investigado, por estarem menos afetados pela interface solo-ar. Essa capacidade dos métodos propostos e as capacidades descritas no Exemplo 1 demonstram, claramente, a importância da contribuição que eles trazem para o conhecimento atual da geofísica aplicada.

\section{Exemplo 4}

Ramirez (1986) empregou a geotomografia eletromagnética para examinar uma região granítica, localizada perto da cidade de Oracle, no Arizona. A distância entre os poços era igual a $30 \mathrm{~m}$. A mesma região foi também investigada por um método acústico de sondagem geofísica que usava poços. Graças a esse método acústico, pôde-se construir uma imagem da distribuição de condutividade hidráulica. Observouse uma correlação genérica entre essa imagem e o tomograma de fator de atenuação. Efetuaram-se também análises de testemunhos geofísicos. Tais análises permitiram que se concluísse que muitas fraturas haviam sido seladas por calcita, o que explicava os valores baixos de condutividade hidráulica e de fator de atenuação, associados a essas fraturas. Um valor de fator de atenuação típico do meio investigado era $0,1 \mathrm{~Np} / \mathrm{m}$. O número de profundidades de penetração é $\alpha L=0,1 \mathrm{~Np} / \mathrm{m}$ X $30 \mathrm{~m}=3$. O topo da região sondada através da geotomografia eletromagnética estava a cerca de $32 \mathrm{~m}$ de profundidade. Pode-se calcular o seno do ângulo de incidência, para ambas as antenas localizadas no topo da região investigada, utilizando-se $h=32 \mathrm{~m}$ e $L=30 \mathrm{~m}$ na Eq.(7). O resultado desse cálculo é $\operatorname{sen} \theta_{i} \cong 0,4244$. Empregando-se tal valor do seno do ângulo de incidência na expressão (11), conclui-se que $\widehat{Q}<0,076$, se a profundidade das antenas não for inferior a $32 \mathrm{~m}$. O valor baixo de $\widehat{Q}$ permite que se afirme que não há praticamente influência da interface solo-ar sobre os dados colhidos, no caso analisado. Ramirez (1986) respalda essa afirmação. A interface solo-ar não causou os artefatos que figuram no tomograma apresentado por Ramirez (1986). Este exemplo ilustra a capacidade que a Eq. (11) possui de auxiliar não só o planejamento de sondagens geotomográficas eletromagnéticas, mas também a interpretação dos geotomogramas. Ademais, o exemplo ilustra a simplificação notável que a Eq. (11) 
traz à análise da influência da interface solo-ar sobre os dados coletados em poços com o radar geofísico.

\section{Exemplo 5}

Grubb et al. (1976) realizaram sondagens geofísicas eletromagnéticas em uma região granítica, localizada perto de Raymond, a cerca de $32 \mathrm{~km}$ a oeste da cidade de Boulder, no Colorado. A distância entre os poços era igual a $90 \mathrm{~m}$. Grubb et al. (1976) notaram, experimentalmente, que os dados coletados até $50 \mathrm{~m}$ de profundidade estavam degradados pela influência da interface solo-ar. Além da sondagem da região localizada entre os poços, efetuada mediante a colocação de uma antena em cada poço, Grubb et al. (1976) também sondaram as cercanias de um dos poços, através da medição da impedância mútua existente entre dois dipolos magnéticos, alinhados paralelamente ao eixo do poço. $O$ valor de condutividade elétrica $(\sigma)$ e o valor de permissividade elétrica relativa $\left(\varepsilon_{r}\right)$, medidos nessa sondagem, foram $\sigma=0,4 \mathrm{mS} / \mathrm{m}$ e $\varepsilon_{r}=9,5$, respectivamente. A freqüência empregada em ambos os tipos de sondagem foi 1,1 MHz. Grubb et al. (1976) omitem o valor do fator de atenuação médio da região localizada entre os poços, bem como o valor do fator de atenuação médio das cercanias do poço onde se efetuaram as medições de impedância mútua. Entretanto, obtém-se uma estimativa do primeiro valor, graças à seguinte equação (Lopes \& Lopes, 1989; Lopes, 1998):

$$
\alpha=\frac{\pi f}{c}\left\{2 \varepsilon_{r}\left[\left(1+\left(\frac{\sigma}{2 \pi \varepsilon_{0} \varepsilon_{r} f}\right)^{2}\right)^{1 / 2}-1\right]\right\}^{1 / 2},
$$

onde $\varepsilon_{0}=8,854185 \mathrm{pF} / \mathrm{m}$ é a permissividade elétrica do espaço livre; $\mathrm{c} \cong 3 \times 10^{8} \mathrm{~m} / \mathrm{s}$ é a velocidade da luz no espaço livre e $f$ é a freqüência. Para os valores de $\sigma, \varepsilon_{r}$ e $f$ supracitados, a Eq.(14) fornece $\alpha=0,023 \mathrm{~Np} / \mathrm{m}$. Para a sondagem efetuada entre os poços, o número estimado de profundidades de penetração é $\alpha L=0,023 \mathrm{~Np} / \mathrm{m} \mathrm{X} 90 \mathrm{~m}=2,1$. Como esse valor se encontra no intervalo $0,5 \leq \alpha L \leq 9,8$, podese empregar a Eq.(13), para determinar a profundidade a partir da qual se garante que a intensidade da onda refletida na interface solo-ar é, no mínimo, dez decibéis inferior à intensidade da onda direta. O resultado desse cálculo é $\hat{h} \cong 50,5 \mathrm{~m}$. Tal valor concorda bastante bem com o valor de profundidade determinado experimentalmente por Grubb et al. (1976), que é $50 \mathrm{~m}$. $\mathrm{O}$ método gráfico e o método numérico também fornecem valores de profundidade próximos de $50 \mathrm{~m}$. Este exemplo ilustra principalmente os seguintes pontos: 1) a validade dos métodos propostos em uma situação prática real; 2) a utilidade desses métodos, pois Grubb et al. (1976) realmente queriam encontrar profundidades em que interferências causadas pela presença de interfaces não prejudicassem a determinação dos parâmetros elétricos constitutivos do meio sondado; 3) a possibilidade de utilização bem-sucedida dos métodos propostos, empregando-se, como estimativa do fator de atenuação da região localizada entre os poços, o valor do fator de atenuação associado às cercanias de um dos poços.

\section{CONCLUSÃO}

Os exemplos práticos reais, analisados aqui, indicaram que a influência da interface solo-ar sobre os sinais captados em poços pode ser avaliada satisfatoriamente pelos métodos desenvolvidos neste trabalho, apesar de eles modelarem, de forma aproximada, as interações complexas existentes entre as ondas eletromagnéticas e o meio geofísico. A capacidade de os métodos superestimarem as condições adversas simplifica o problema, amplia a utilidade deles e favorece a obtenção de resultados com margem de segurança. Podem-se emitir ondas TE, de um poço, empregando-se uma antena com uma fenda orientada axialmente em um cilindro condutor. Se tais ondas forem empregadas, ainda assim os três métodos apresentados neste trabalho podem ser utilizados, pois a Eq.(5) não envolve o coeficiente de reflexão, o qual depende do tipo de polarização. O uso de ondas TE e TM, para sondar uma mesma região, permite caracterizar melhor os alvos. Se o radar geofísico de poços empregar ambas essas ondas, ele pode ser qualificado como polarimétrico. Além do dipolo elétrico, que é convencionalmente usado em sondagens efetuadas com o radar de poços, uma antena com fenda também pode emitir ondas TM. Evidência a esse respeito é fornecida por Lopes (1985), que projetou, construiu e testou uma antena com uma fenda disposta ao longo da circunferência de um cilindro de cobre. Miwa et al. (1999) demonstram, através de medições reais, que um dipolo elétrico e uma antena com uma fenda orientada axialmente em um cilindro condutor podem apresentar diagramas de radiação praticamente iguais ao longo do plano de incidência. O Princípio de 
Babinet (Ramo et al., 1965) explica o porquê dessa semelhança. É graças a ela que se pode afirmar que os três métodos desenvolvidos neste trabalho para as ondas TM, emitidas pelo dipolo elétrico, também se aplicam às ondas TE, emitidas pela antena com fenda orientada axialmente.

Embora a validade dos três métodos apresentados neste trabalho tenha sido verificada em diversas sondagens eletromagnéticas, efetuadas com o emprego de poços, apresentaram-se apenas cinco exemplos da utilização dos métodos, por motivo de concisão. Entretanto, os exemplos foram judiciosamente escolhidos, com o intuito de ilustrar, de forma tão pragmática quanto possível, a adequação dos métodos a meios geofísicos diversificados. Os métodos desenvolvidos podem aproveitar medidas das características elétricas específicas de cada meio geofísico. Destarte, pode-se dispensar a utilização de valores tabelados para materiais de constituição e propriedades físico-químicas amplas. A caracterização constitucional de tais materiais compostos costuma ser difícil de ser executada. A excelente concordância entre as previsões efetuadas pelos métodos desenvolvidos e os resultados experimentais, relatados na literatura internacional, é uma evidência significativa de que os métodos são bastante confiáveis. Os exemplos práticos reais investigados ressaltam a importância desses métodos tanto para o planejamento de sondagens eletromagnéticas, realizadas com o uso de poços, como para a interpretação dos resultados de tais sondagens.

\section{AGRADECIMENTOS}

Os autores agradecem as colaborações prestadas pelo Dr. Augusto Cesar Pires, Consultor do Senado Federal, e pelo Dr. Luiz Muniz Barreto, Pesquisador do Departamento de Geofísica do Observatório Nacional.

\section{REFERENCIAS}

ALEXANDER, M.J. \& SALTER, M.J. -1997- The design of dipole and monopole antennas with low uncertainties. IEEE Transactions on Instrumentation and Measurement, 46: 539-543.

ALUMBAUGH, D.L. \& NEWMAN, G.A. -1997Three-dimensional massively parallel electromagnetic inversion. Part II. Analysis of a crosswell electromagnetic experiment. Geophysical Journal International, 128: 355-363.

CôTe, P., DEGAUQUE, P., LAGABRIELLE, R. \& LEVENT, N. -1995- Detection of underground cavities with monofrequency electromagnetic tomography between boreholes in the frequency range $100 \mathrm{MHz}$ to $1 \mathrm{GHz}$. Geophysical Prospecting, 43: 1083-1107.

ELLIS, G.A. \& PEDEN, I.C. -1997- Cross-borehole sensing: identification and localization of underground tunnels in the presence of a horizontal stratification. IEEE Transactions on Geoscience and Remote Sensing, 35: 756-761.

GRUBB, R.N., ORSWELL, P.L. \& TAYLOR, J.H. -1976- Borehole measurements of conductivity and dielectric constant in the $300 \mathrm{kHz}$ to $25 \mathrm{MHz}$ frequency range. Radio Science, 11: 275-283.

HANSEN, T.B. \& JOHANSEN, P.M. -2000Inversion scheme for ground penetrating radar that takes into account the planar air-soil interface. IEEE Transactions on Geoscience and Remote Sensing, 38: 496-506.

KING, R.W.P. \& BROWN, M.F. -1984- Lateral electromagnetic waves along plane boundaries: a summarizing approach. Proceedings of the IEEE, 72: 595-611.

KING, R.W.P. \& SANDLER, S.S. -1994- The electromagnetic field of a vertical electric dipole over the earth or sea. IEEE Transactions on Antennas and Propagation, 42: 382-389.

KRAUS, J.D. -1992- Electromagnetics, McGraw-Hill, Singapore, 847pp.

LOPES, ELIZEU P. -1985- Antena Slot Cilíndrica. Projeto final de graduação, Departamento de Engenharia Eletrônica, UFRJ.

LOPES, ELIEZER P. -1989- Tomografia geofísica eletromagnética. Dissertação de mestrado, COPPE/UFRJ.

LOPES, ELIEZER P. -1998- Geotomografia eletromagnética: algoritmo tomográfico, filtro bidimensional adaptativo e algoritmo de segmentação de imagens. Tese de doutorado, UFSC.

LOPES, ELIEZER P. \& LOPES, ELIZEU P. 1989- Tomografia geofísica eletromagnética. Anais VII Simpósio Brasileiro de Telecomunicações, 1: 539-545, Florianópolis, SC.

LOPES, ELIEZER P. \& LOPES, ELIZEU P. 1992- Electromagnetic geophysical tomography. IEE Proceedings F - Radar and Signal Processing, 139: $27-35$

LOPES, ELIEZER P. \& LOPES, ELIZEU P. 1993- Um novo algoritmo paralelo para a reconstrução de imagens de meios geofísicos. Anais XI Simpósio Brasileiro de Telecomunicações, 
1: 165-170, Natal, RN.

LOPES, ELIEZER P. \& LOPES, ELIZEU P. 1994- Geotomografia eletromagnética. Revista Brasileira de Geofísica, 12: 163-175.

LOPES, ELIEZER P., LOPES, ELIZEU P., BARRETO, J.M. \& BARRETO, L.M. -1998Segmentation tomography. Electronics Letters, 34: 2222-2224.

LYTLE, R.J., LAINE, E.F., LAGER, D.L. \& OKADA, J.T. -1976- Determination of the in situ high frequency electrical properties of permafrost rock. Radio Science, 11: 285-293.

MIWA, T., SATO, M. \& NIITSUMA, H. -1999Subsurface fracture measurement with polarimetric borehole radar. IEEE Transactions on Geoscience and Remote Sensing, 37: 828-837.

RAMIREZ, A.L. -1986- Recent experiments using geophysical tomography in fractured granite. Proceedings of the IEEE, 74: 347-352.
RAMO, S., WHINNERY, J.R. \& DUZER, T.V. 1965- Fields and Waves in Communication Electronics, Wiley, New York, 754pp.

SADIKU, M.N. -1994- Elements of Electromagnetics, Sanders, Forth Worth, 821pp.

SHAVIT, R. \& ROSEN, E. -1995- Lateral wave contribution to the radiation from a dielectric half medium. IEEE Transactions on Antennas and Propagation, 43: 751-755.

TURNER, G. \& SIGGINS, A.F. -1994- Constant $Q$ attenuation of subsurface radar pulses. Geophysics, 59: 1192-1200.

VESECKY, J. -1999- San Diego HF radar user workshop'99. Backscatter, 10: 15-25.

YAMAGUCHI, Y., NISHIKAWA, T., SENGOKU, M. \& BOERNER, W. -1995- Two-dimensional and full polarimetric imaging by a synthetic aperture FM-CW radar. IEEE Transactions on Geoscience and Remote Sensing, 33: 421-427.

\section{ASSESSING THE INFLUENCE OF THE GROUND-AIR INTERFACE ON POLARIMETRIC BOREHOLE-RADAR DATA}

The first georadar survey was carried out in Austria in 1929. The survey aimed at discovering the depth of a glacier. In the late 1950s, planes trying to land in Greenland crashed into the ice because the ability of radar waves to penetrate ice degraded altitude information. The accidents spurred researches into radar soundings of subsurface structures. Subsurface radar surveys can be performed from the earth's surface, aircraft, satellites or wells. Antennas near the ground-air interface can transmit or receive electromagnetic waves that are closely coupled to it. Such waves are called "lateral waves". If the transmitting and receiving antennas are at shallow depths, then the wave reflected at the ground-air interface effectively cancels the direct wave, and the received signal is mainly due to the lateral wave. As the depth of both antennas increases, the direct and reflected waves no longer effectively cancel each other, and their influence on the received signals becomes significant. As the depth of the antennas increases even more, the contribution of the lateral wave to the received signals tends to become negligible.

Some authors of articles on borehole radar recommend that the distances between the ground-air interface and the observation points be large to prevent signals associated with direct waves from being degraded by signals concerning waves scattered at the interface. But what is the criterion used to decide that a given distance is large enough? In a practical example studied in this paper, a depth of nearly $91 \mathrm{~m}$ in a limestone region meets the distance requirement. In another example, which addresses an actual subsurface-radar survey performed in a granitic formation, a depth of only $6.1 \mathrm{~m}$ meets the distance requirement. All the examples analyzed in this paper involve data collected in actual borehole-radar surveys. The experimental depth values closely match the values calculated by a numerical method, an analytical method and a graphical method that are proposed in this work.

To the authors' knowledge, no tools are available to quantitatively assess the influence of the ground-air interface on borehole-radar data before initiating the datalogging process. In typical borehole-radar surveys, data that are deemed to have been scattered by the ground-air interface are usually discarded after analysis of the received signals. Automating the analysis process is a difficult task mainly when continuous-wave excitation is employed. Attempts to delegate the analysis process to artificial neural networks have led to unsatisfactory results owing to the diversity of situations involved in the problem: characteristics of the media, distances between boreholes, excitation modes, data kinds (amplitude data, phase data or travel-time data), etc. Since the underground environment may be complex, lack of theoretical support may render the field survey unprofitable or even meaningless. The methods devised herein provide 
theoretical support to the aforementioned data-analysis problem.

Eq.(13) expresses the depth where the intensity of the direct-arriving signal is at least $10 \mathrm{~dB}$ greater than the intensity of the signal arriving at the receiving antenna after reflecting at the ground-air interface. The reader interested in arbitrary ratios between the intensity of the reflected signal and the intensity of the direct-arriving signal may resort to the graphical method employed in Example 1. Fig.3 reveals that this method cannot be used if the number of penetration depths $(\alpha \mathrm{L})$ exceeds 6. The analytical method described by Eq.(13) cannot be employed if $\widehat{Q} \neq 0.1$ or the number of penetration depths does not belong to the range $0,5 \leq \alpha L \leq 9,8 . \widehat{Q}$ is the ratio between the magnitudes of the reflected and direct electric fields, measured by the receiving antenna in a vertical borehole, assuming total reflection at the ground-air interface. This assumption simplifies the problem, usually enables calculation of the antenna depth with a safety margin, and makes the methods devised applicable to both the TM polarization and the TE polarization. When neither the graphical method nor the analytical method can be used, Eq.(5) must be solved numerically for $\sin \theta_{i}$, where $\theta_{i}$ is the angle of incidence shown in Fig.2. The computed value of $\sin \theta_{i}$ is converted into a depth value by means of Eq.(6). Analyses of Eq.(5) and Fig.3 indicate that $\hat{Q}^{1 / 3}<\sin \theta_{i}<1$ for a buried receiving antenna. Knowledge of this fact is useful to set the starting value of $\sin \theta_{i}$, which is required to numerically solve Eq.(5).

A sensitivity analysis of $\hat{Q}$ to the average attenuation factor $(\alpha)$ of the interborehole region demonstrates that $\bar{Q}$ varies inversely with $\alpha$. This means that underestimating $\alpha$ causes not only the intensity of the reflected signal to be overestimated but also the calculated minimum depth of the antennas to be overvalued. It is therefore safer to underestimate $\alpha$ when there is much uncertainty about its value. This conclusion also applies to polarimetric borehole radars. Most borehole radars employ electric dipoles as transmitting and receiving antennas. In a borehole, lack of room often prevents an electric dipole from being widely rotated around any axis whose orientation differs from that of the borehole. As a result, the majority of borehole-radar systems do not perform polarimetric measurements. However, a system made up of a pair of transmitting antennas and a pair of receiving antennas can make such measurements. Each pair comprises an axial slot on a conducting cylinder and the usual electric dipole antenna. The former antenna emits waves whose polarization is perpendicular to the borehole axis. Compared to the single-polarization radar, the polarimetric radar enables the extraction of more information out of the targets, thereby making their imaging and characterization easier.

\section{NOTE ABOUT THE AUTHORS}

\section{Eliezer Pereira Lopes}

Graduou-se em Engenharia de Sistemas e Computação, na Universidade do Estado do Rio de Janeiro (UERJ), e recebeu o título de Mestre em Ciências em Engenharia Elétrica, em 1989, com a dissertação intitulada Tomografia Geofísica Eletromagnética, defendida na Coordenação de Programas de Pós-Graduação em Engenharia (COPPE) da Universidade Federal do Rio de Janeiro (UFRJ). Ele possui especialização em Telecomunicações, realizada na UFRJ; Proficiência em Língua Inglesa, concedida pela Universidade de Cambridge, na Inglaterra; Licenciatura em Língua Inglesa, conferida pela UERJ; e Doutorado em Engenharia Elétrica, realizado na Universidade Federal de Santa Catarina, onde defendeu a tese intitulada Geotomografia Eletromagnética: Algoritmo Tomográfico, Filtro Bidimensional Adaptativo e Algoritmo de Segmentação de Imagens. É o introdutor da Geotomografia Eletromagnética no Brasil, e autor de várias publicações no Brasil e no exterior. Tem participado de várias conferências nacionais e internacionais. Em 1998, ele foi apontado para integrar a Academia de Ciências de Nova Iorque e para figurar no Who is Who in the World. Suas áreas de atuação e de maior interesse são: Computação Aplicada; Redes Neurais; Inteligência Artificial; Geoprocessamento; Matemática Computacional; Processamento Paralelo; Problemas Inversos Biomédicos e Geofísicos; Radares de Subsuperfície, Oceânico e Meteorológico; Coleta Automática de Dados Meteorológicos e Hidrológicos; Codificação e Transmissão de Sinais Multidimensionais; Processamento de Imagens e de Sinais; Antenas; Telemática; Propagação de Ondas; Fibras Óticas; Telecomunicações; Ensino de Informática e Ensino da Língua Inglesa.

\section{Elizeu Pereira Lopes}

Graduou-se com louvor em Engenharia Eletrônica, na Universidade Federal do Rio de Janeiro (UFRJ), e obteve o título de Mestre em Ciências em Engenharia Elétrica, com a dissertação intitulada Métodos de Reconstrução de Imagens, defendida, em 1989, na Coordenação de Programas de Pós-Graduação em Engenharia (COPPE) da UFRJ. Possui Proficiência em Língua Inglesa, concedida pela Universidade de Cambridge, na Inglaterra; Licenciatura em Língua Inglesa, conferida pela Universidade do Estado do Rio de Janeiro; e Doutorado em Engenharia Elétrica, realizado na Universidade Federal de Santa Catarina, onde defendeu a tese intitulada Sondagem de Meios Estratificados com o Radar de Subsuperficie Neural. Ele é autor de várias publicações nacionais e internacionais. Tem sido convidado para ministrar várias palestras em instituições nacionais. É pioneiro, no Brasil, no desenvolvimento do Radar de Subsuperfície Neural. Suas áreas de maior interesse são: Processamento de Imagens, Sensoriamento Remoto, Redes Neurais, Eletromagnetismo, Geofísica Pura e Aplicada, Análise Espacial, Controle de Processos por Computador, História da Ciência, Comunicação de Dados, Comunicações Óticas e Comunicações por Satélite. 